\title{
Un latido más de avance para la salud en el Tolima
}

\section{One more heartbeat of progress for health in Tolima}

\section{Valentina Cárdenas Cadena*}

O

Recepción: 04/11/2021
Aprobación: 26/11/2021

Publicación: 22/12/2021

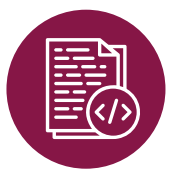

Para citar este artículo

Cárdenas Cadena, V. (2021). Un latido más de avance para la salud en el Tolima. Indagare, (9), 103-108.

https://doi.org/10.35707/indagare/911

\footnotetext{
1 " Programa de Comunicación Social y Periodismo de la Universidad de Ibagué, Ibagué, Colombia.
} ORCID: 0000-0001-8569-6745. Correo electrónico: cardenas.valentina0@gmail.com 


\section{Resumen}

El Ministerio de Ciencia, Tecnología e Información (Minciencias), en el marco de su Convocatoria 896, otorgó el financiamiento para el desarrollo del proyecto "Biodiseño: una oportunidad para mejorar el manejo de la enfermedad cardiovascular en el Tolima", liderado por dos grupos de investigación de la Universidad de Ibagué (2021): D+TEC y GMAE, con el apoyo del grupo de ingeniería biomédica de la Universidad de los Andes. Este proyecto que se destaca por su innovación tiene un sentido más trascendental, pues pretende ser una alternativa para darle manejo a una de las enfermedades que más ha arrebatado vidas en los últimos años.

\section{Abstract}

The Ministry of Science, Technology and Information (Minciencias), within the framework of its Call 896, granted funding for the development of the project "Biodesign: an opportunity to improve the management of cardiovascular disease in Tolima," led by two research groups of Universidad de Ibagué (2021): D+TEC and GMAE, with the support of the biomedical engineering group of Universidad de los Andes. This project, which stands out for its innovation, has a more transcendental meaning, as it intends to be an alternative to manage one of the diseases that has taken the most lives in recent years.

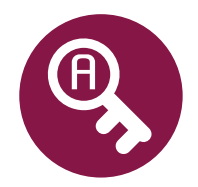

Palabras claves: salud, enfermedades cardiovasculares, financiación, biodiseño, medicina.

Key words: Health, cardiovascular disease, financing, biodesign, medicine.

El proyecto "Biodiseño: una oportunidad para mejorar el manejo de la enfermedad cardiovascular en el Tolima" surge a raíz de la Convocatoria 896: "Fortalecimiento de capacidades regionales de investigación en salud" del Ministerio de Ciencia, Tecnología e Innovación (Minciencias), que tiene como objetivo contribuir a la solución de los principales problemas y necesidades de salud en las regiones (Amazonía, Andina, Caribe, Orinoquía y Pacífica).

El departamento del Tolima está priorizado en los términos de referencia por el bajo índice de desarrollo que presenta, y los grupos de investigación: D+TEC y GMAE de la Universidad de Ibagué decidieron participar en esta convocatoria logrando satisfactoriamente una financiación y la oportunidad de ejecutar un proyecto que podría cambiar la vida de cientos de tolimenses. 
La revista Indagare tuvo la oportunidad de conversar con la participante de este proyecto, docente de la Universidad de Ibagué, y en la actualidad adscrita al grupo de investigación GMAE: Luz Adriana Sánchez Echeverri, ingeniera física, con magíster en Ciencia e Ingeniería y doctora en Ingeniería, quien adicionalmente ha tenido la oportunidad de desarrollar proyectos de investigación de financiación interna e internacional enfocados en la producción, caracterización y desarrollo de nuevos productos.

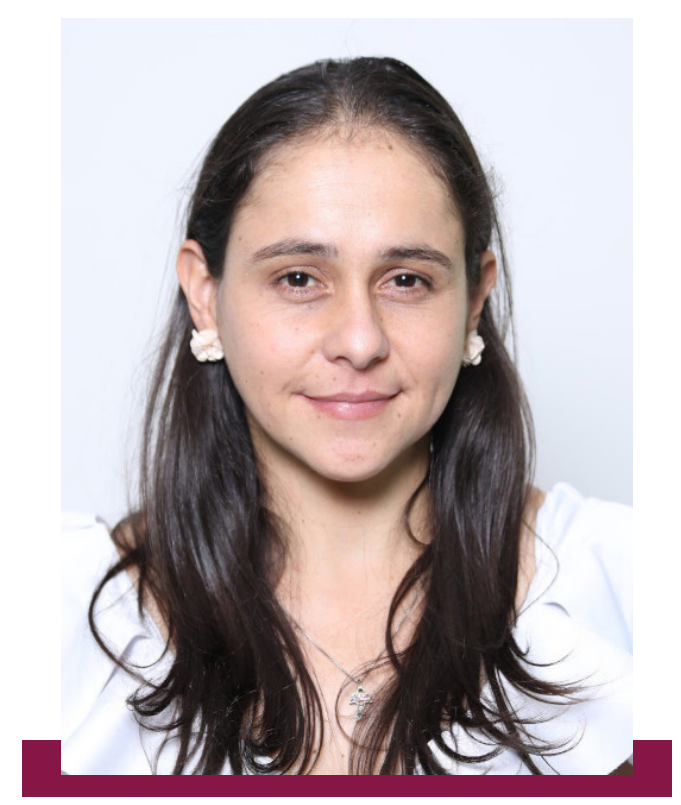

Fuente: Comunicación institucional, Universidad de Ibagué

\section{¿Por qué se enfocaron en las enfermedades cardiovasculares?}

Una de las líneas priorizadas en la convocatoria eran las Enfermedades Crónicas No Transmisibles (ENT). El término hace referencia a un grupo de enfermedades de larga duración y de necesidad de tratamiento a largo plazo. Dentro de estas, se encuentran las enfermedades cardiovasculares (Organización Mundial de la Salud, 13 de abril de 2021).

Del grupo de las enfermedades crónicas no transmisibles, las enfermedades cardiovasculares tienen un mayor índice de mortalidad. Además, el departamento del 
Tolima es una de las regiones con mayor índice de envejecimiento, lo cual genera una alta predisposición a la enfermedad cardiovascular.

\section{¿Quiénes hacen parte del proyecto?}

Nuestro primer aliado es el grupo de ingeniería biomédica de la Universidad de los Andes (GIB-UA), el que ya ha tenido experiencia en la implementación de la metodología de biodiseño en la investigación de la dinámica cardiovascular, y en el desarrollo de dispositivos médicos.

Por parte de la Universidad de Ibagué, somos cuatro profesores vinculados en este proyecto. Está el grupo de investigación D+TEC, en el que participan los docentes Oscar Javier Araque (ingeniería mecánica), Luisa Fernanda Gallo (ingeniería electrónica) y Jorge Andrés García (ingeniería mecánica). Por otro lado, se encuentra el grupo de investigación GMAE, en el cual participo. Como grupos de investigación hemos logrado avances en el análisis de imágenes para patologías cardiovasculares, y en el desarrollo de prototipos mecánicos y electrónicos.

\section{En términos generales, ¿en qué consiste el proyecto?}

Tenemos un primer objetivo que es el diagnóstico. El equipo investigador junto con el equipo médico, que en esta oportunidad nos acompaña la Clínica Medicadiz, buscamos entender: ¿Cuáles son las necesidades prioritarias que se derivan de este tipo de enfermedades? Ya sea en el diagnóstico o en el tratamiento.

Una vez identificadas estas necesidades, se hace un listado de alternativas. Nosotros dentro del equipo de investigación, en compañía de los médicos, podemos revisar qué alternativas pueden implementarse para dar solución a estas necesidades. Cuando hablamos de alternativas nos referimos a un dispositivo, una aplicación, una metodología, un programa.

Esta metodología tiene la característica que no es que diseñemos el dispositivo, sino que evaluamos ese listado de alternativas. No necesariamente las tenemos que inventar, porque para eso existe la revisión de literatura. Esta última consiste en mirar qué se ha hecho en otros países. Revisamos los requerimientos técnicos, financieros, y que sean alternativas que se puedan aplicar en el contexto regional.

También se revisa el grado de transferencia que puedan tener: que sea una alternativa fácil de implementar no solo en la Clínica Medicadiz, sino también en los diferentes sectores de la salud que presenten estas problemáticas.

Se evalúan esos requerimientos para aplicar esas alternativas, y nuevamente se realiza una priorización para saber cuál es la alternativa más viable para desarrollar en cuanto a tiempo, recursos, y otros aspectos. Después de dicha priorización, se efectúa el desarrollo del prototipado, y se implementan al menos dos (2) alternativas. 
Al final, el proyecto pretende que esas dos alternativas que se están priorizando, sean implementadas y tengan las capacidades de ser transferidas, no solo en Ibagué, sino en el Tolima.

\section{¿Qué beneficios se logran con la implementación de este proyecto?}

Darle una oportunidad o una mejora en el manejo de estas enfermedades. De igual forma, nosotros también queremos que se cree una estrategia de transferencia. Que no solo se quede en la ejecución, sino que nuestros resultados también logren llegar a diferentes instituciones.

Adicionalmente, para la Universidad de Ibagué se fortalecerán los laboratorios, porque contaremos con unas impresoras de gran formato: una 3D y un equipo de caracterización de materiales, derivados de este proyecto.

Y en una perspectiva personal, el gran beneficio es la experiencia. Tanto en la metodología que estamos aplicando como en la formulación de proyectos. Es algo que a mí y al equipo investigador nos va a servir mucho, y al lograr esta experiencia será mucho más fácil entrar a otras convocatorias.

\section{¿Cómo recibió el grupo de investigación la noticia de que el proyecto fue elegible para financiación?}

Muy contentos. Hoy en día, las universidades están buscando recursos externos para la financiación de proyectos, porque en algún momento no se vuelve sostenible estar financiando desde la misma universidad todos los proyectos. Ese es el modelo actual de las grandes universidades.

Muchas veces nos da miedo participar, salir de la zona de confort. Uno cree que no, pero nosotros tenemos todas las capacidades para ser financiados de manera externa. La gran mayoría de profesores hemos participado en convocatorias internas, y ya tenemos esa experiencia de escribir. Es soltar ese miedo y decir "sí, vamos a postularnos en convocatorias externas". Una vez se realiza por primera vez, uno se da cuenta que no es tan difícil ni imposible de hacer.

Ahora que ya pasa la alegría de saber que nos ganamos el proyecto, empieza el momento de ejecutar. 
La financiación de este proyecto es una muestra del talento humano y la alta calidad de docentes e investigadores con los que cuenta la Universidad de Ibagué. Estos resultados son una invitación a la comunidad universitaria para postularse a este tipo de convocatorias, pues aunque trae beneficios significativos para las universidades partícipes, docentes y demás involucrados, todo se resume en la satisfacción de poder sumar al desarrollo de nuestra región e impactar a un grupo poblacional que merece los mayores esfuerzos de nuestra sociedad.

\section{Referencias}

Organización Mundial de la Salud. (13 de abril de 2021). Enfermedades no transmisibles. Recuperado de https://www.who.int/es/news-room/fact-sheets/detail/noncommunicable-diseases

Universidad de Ibagué. (2021). Biodiseño: una oportunidad para mejorar el manejo de la enfermedad cardiovascular en el Tolima. (Informe ejecutivo). Ibagué, Colombia: Universidad de Ibagué. 